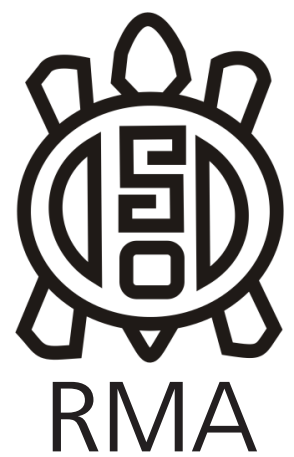

Arqueología

\title{
La elección de soportes laminares a lo largo del Holoceno en el área Interserrana Bonaerense (Argentina)
}

\author{
The choice of laminated supports throughout the Holocene in the \\ Interserrana Bonaerense area (Argentina) \\ María Paula Barros
}

Instituto de Investigaciones Arqueológicas y Paleontológicas del Cuaternario PampeanoConsejo Nacional de Investigaciones Científicas y Técnicas (INCUAPA-CONICET). Facultad de

Ciencias Sociales, Universidad Nacional del Centro de la Provincia de Buenos Aires. Av. Del Valle 5737, Olavarría (B7400JWI), Buenos Aires, Argentina. E-mail: mapaubarros@gmail.com

\begin{abstract}
Resumen
En este trabajo se presenta el análisis y la discusión acerca de la producción de módulos laminares sobre dos rocas ampliamente utilizadas en la subregión pampa húmeda, como son la ortocuarcita del Grupo Sierras Bayas y la ftanita. Los datos provienen de diferentes sitios arqueológicos del área Interserrana (región pampeana bonaerense) y sus cronologías representan distintos momentos del Holoceno. Este tipo de producción se presenta en bajos porcentajes, si se compara con un tipo de extracción de lascas más anchas y cortas. Sin embargo, se considera importante estudiar las condiciones de su elección, siendo que permite generar soportes con filos independientes de diferentes largos, propiciando que un mismo artefacto pueda ser utilizado para la ejecución de una variedad de tareas. En este sentido, se busca indagar acerca de cuáles fueron los criterios técnicos tenidos en cuenta, tanto para la preparación de los núcleos, como para su explotación. Se consideran distintas variables, como materia prima lítica, modo de aprovisionamiento, y objetivo de producción, las cuales jugaron un rol significativo a la hora elegir este tipo de extracción.
\end{abstract}

Palabras clave: área Interserrana; tecnología lítica; objetivos de producción; módulo laminar.

\begin{abstract}
This article presents the results and discussion on the production of laminar technology on two broadly used raw materials from the Humid pampas: orthoquarzite from Grupo Sierras Bayas and ftanite. The data come from different archaeological sites of the Interserrana area; these contexts are dated to different periods of the Holocene. When compared with the extraction of wider and shorter flakes, the blades are usually represented with lower percentages. Nevertheless, it is important to study the circumstance sin which blade technology was selected, because this technology allows the obtaining of blanks with independent edges of different length; thus, a single artifact could have been used to develop different tasks. In this sense, one of the aims of this work is to determine the technical criteria that were considered for the preparation and exploitation of the cores. Finally, different variables, such as raw material, acquisition, and production objective, played a significant role at the moment of choosing this kind of extraction.
\end{abstract}

Keywords: Interserrana area; lithic technology; objectives of production; laminar technology.

En este trabajo se realiza un estudio de la producción de módulos laminares ${ }^{1}$ confeccionados sobre ortocuarcita del Grupo Sierras Bayas (GSB) y ftanita, tomando en consideración el material proveniente de distintos sitios arqueológicos Paso Otero 4 (partido de Necochea), Laguna

\footnotetext{
${ }^{1}$ En este trabajo se utilizan los conceptos de Producción de módulos laminares, Hoja y Lámina, los cuales se definen de la siguiente forma: Producción de módulos laminares: Se refiere a núcleos que presenten negativos de extracciones laminares, producción de soportes con módulo laminar y desechos que presenten módulo laminar. En relación a Hoja y Lámina se sigue la propuesta realizado por Aschero 1975, Hojas: lascas de longitud variable (menor o mayor a dos anchos), bordes (laterales) paralelos o subparalelos, talones lisos, diedros, facetados y puntiformes. Lámina: lasca de longitud igual o mayor a dos anchos, bordes subparalelos, cara dorsal con reserva de corteza, con aristas de lascados angulares o con arista única longitudinal, talones lisos-naturales, lisos, diedros y facetados.
}

Muscar 2 y Laguna Seca 2 (partido de General Lamadrid) y Alero La Virgen (partido de Olavarría). Asimismo, se incluirán datos del análisis de una muestra del material que fue recolectado en el área del río Quequén Grande, específicamente en la localidad arqueológica Zanjón Seco y que actualmente se encuentra en el Museo de Ciencias Naturales "Gesué P. Noseda" del Club de Pesca de Lobería (partido de Lobería). Si bien el uso de soportes laminares ha sido documentado en diferentes períodos cronológicos, es necesario realizar un análisis comparativo entre diferentes sitios con el fin de establecer si la elección de este tipo de soporte está ligada a una continuidad tecnológica regional a lo largo del Holoceno.

Las primeras referencias acerca de la presencia de 
producción laminar y de hojas en el área Interserrana y zonas aledañas provienen de Madrazo (1972, 1973), quien la identifica en diferentes sitios arqueológicos superficiales de la zona de Lobería, Quequén Chico y Tandil. Con respecto a la cronología, Madrazo (1979) consideró que la producción laminar tenía una antigüedad considerable aunque no especifica una fecha absoluta.

Posteriormente, y ya en el marco del estudio de la organización tecnológica, continúan las menciones de producción laminar en diferentes sitios de la región pampeana. Sin embargo, dichas menciones se limitan a señalar su presencia o ausencia en los sitios estudiados, dejando de lado la discusión acerca de las ventajas o desventajas de la implementación de este tipo de tecnología. Un ejemplo de ello es el estudio efectuado por Nami (1999), donde analiza láminas provenientes de la colección de superficie de Michelutti que se encuentra en Púan. También en el sitio Paso Otero 4 se ha propuesto que existe una tendencia laminar presente en los niveles inferiores (ca. 8900 a 7700 años AP) (Barros et al. 2014). En el sitio multicomponente El Guanaco, ubicado en la llanura pampeana, hay un amplio predominio de talla unifacial y una alta frecuencia de bipolaridad, aunque también se registraron bifaces y algunas hojas para las ocupaciones del Holoceno tardío. Estas últimas pudieron ser obtenidas a través de la explotación de núcleos atípicos (Bayón y Flegenheimer 2004). Por otra parte, en el área de las canteras del Arroyo Diamante, hay registros de hojas y las mismas provienen de la producción efectuada a partir de núcleos piramidales (Flegenheimer et al. 1996).

\section{Marco teórico y metodológico}

Para el análisis de los materiales de los distintos sitios se utiliza una perspectiva tecno-tipológica, siguiendo autores como Aschero (1975, 1983); Bellelli et al. (1985-87),
Boëda et al. (1990), Boëda (1994, 1997), Inizan et al. (1995); Perlès (1991), Pelegrin (1995), entre otros. Para el análisis de los modos de producción y de los núcleos se sigue el concepto de cadena operativa, el cual es utilizado por distintas corrientes dentro de la arqueología francesa. En este trabajo seguimos la definición propuesta por Geneste (1991) donde la cadena es dividida en cinco fases sucesivas: 1- aprovisionamiento de materias primas; 2-transformación y preparación de la materia prima en bruto, talla inicial; 3- talla de productos brutos, obtención de las formas base; 4- transformación de los soportes en instrumentos (retoque o formatización) y 5- utilización y desgaste de instrumentos (mantenimiento y reutilización). Por último se llega al descarte final del instrumento. Asimismo, se emplea la metodología planteada por el principio estructural de los modos de producción (Boëda 2005; Fogaça y Boëda 2006). Este se divide en estructura adicional, donde se incluyen los sistemas, A, B, C y D y estructura integrada, compuesta por los sistemas $E$ y F. Todos los sistemas son capaces de responder a una demanda de instrumentos cada vez mas estructurados. A continuación se resumen las principales características.

\section{Estructura adicional}

El nódulo o bloque (sensu Aschero 1975) a explotar puede ser dividido en dos subconjuntos, uno no operacional y otro operacional; este último es denominado núcleo (Figura II). Esta estructura se divide en cuatro sistemas, A, $B, C$ y D, las cuales se aplican al subconjunto operacional.

Sistema A: Se trata de la búsqueda de un filo. No hay ninguna predeterminación, solo la parte transformativa es buscada. Es decir la zona del artefacto que se va a poner contacto con la materia a transformar. Toda lasca presenta un filo que puede ser natural o deberá realizarse su transformación por medio del retoque. El soporte

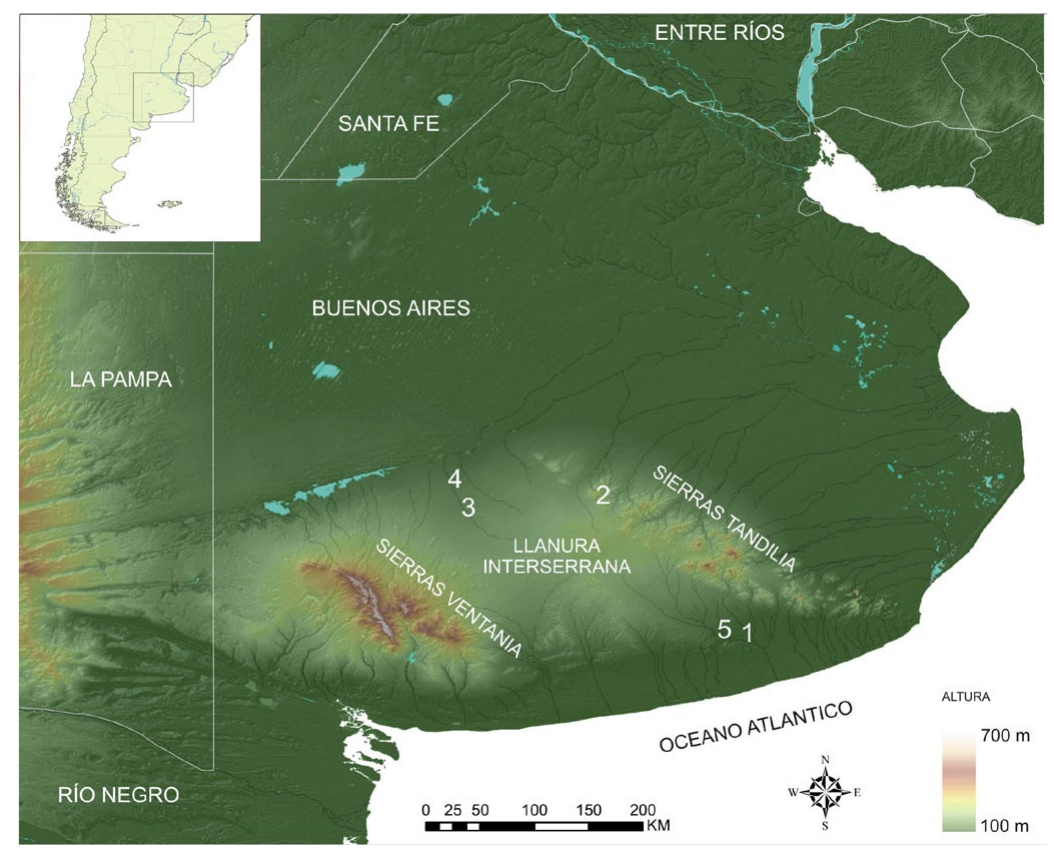

Figura 1. Ubicación de los sitios analizados en la subregión Pampa Húmeda. Referencias: 1- Sitio Paso Otero 4. 2- Sitio Alero La Virgen. 3-Sitio Laguna Muscar 2. 4- Laguna Seca 2. 5- Área de la localidad arqueológica Zanjón Seco, cuyo material se encuentra depositado en el Museo de Ciencias Naturales "Gesué P. Noseda" del Club de Pesca de Lobería (partido de Lobería)

Figure 1. Location of the analysed sites in the Humid Pampa Subregion. References:: 1- Paso Otero 4 site. 2-Alero La Virgen site. 3-Laguna Muscar 2 site. 4-Laguna Seca 2 site. 5- Area of the archaeological locality Zanjón Seco, which material is despoisted in the Museum of Natural Sciences "Gesué P. Noseda", of the Fishermen Club from Lobería (Lobería district) 

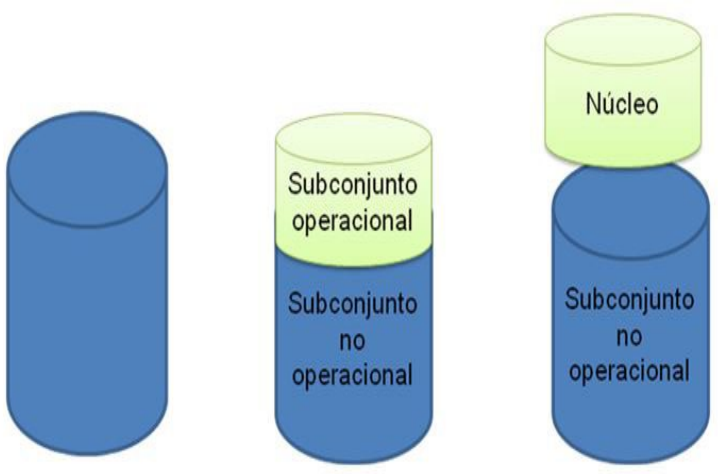

Figura 2. Esquema de la estructura adicional

Figure 2. Aditional structure scheme

puede ser recolectado de la superficie o extraído de un bloque o nódulo.

Sistema B: Producción de más de un filo. Adopción de la noción de recurrencia para realizar extracciones sucesivas permitiendo el aumento de características del filo, como su regularidad y delineación.

La noción de recurrencia designa una relación de causalidad, definida en que, una vez terminada la secuencia se vuelve al punto de partida (Boëda 1997). Toda extracción proveniente de una misma serie recurrente está en relación a extracciones anteriores y son denominadas extracciones predeterminadas. Mientras que las extracciones predeterminantes crean nuevos criterios técnicos luego de cada extracción (Boëda 1997).

Sistema C: Es una producción de una o varias extracciones con un control sobre la morfología. Se explota un subvolumen de tipo $C$, con características de convexidad presentes de manera natural. Se selecciona el bloque o nódulo por su morfología.

Características del sistema C:

-Para un objetivo particular, se busca una convexidad natural particular sobre un bloque.

-Las extracciones que siguen, utilizan las aristas y tienen una morfología más variable.

-Todas las formas pueden obtenerse ya que hay una selección y una elección de la superficie a reducir en función de los objetivos.

-Las secuencias de débitage son relativamente cortas.

Tipo C 1: laminar, búsqueda de lascas más largas que anchas.

Tipo C 2: con búsqueda de lascas triangulares, no es común ya que la convergencia de los dos bordes puede también ser obtenida por el retoque o la confección.

Sistema D: Se encuentra el nódulo o bloque natural, luego, se inicia la preparación del futuro núcleo, creándose las convexidades. Posteriormente se comienza a explotar el núcleo y se extraen lascas.
Características del sistema D

El objetivo no se inscribe en la morfología del bloque (tipo C), y requiere una fase de adaptación previa (preparación de la estructura del bloque).

El sistema D permite obtener todas las formas de lascas, como alargadas, triangulares, cuadradas y se detiene cuando la estructura establecida se agota, aunque puede también detenerse en curso de la explotación ya que se lograron los objetivos.

\section{Estructura integrada}

El nódulo o bloque a explotar siguió un proceso de inicialización, creando una nueva entidad (Figura III). En esta categoría estructural, el conjunto operacional se confunde con la integralidad del volumen del bloque a explotar, es decir, agrupa los sistemas técnicos de producción que necesita el bloque íntegro para realizar sus objetivos. Están conformados en un mismo sistema de sinergia de producción y se divide en dos sistemas, E y F.

Sistema E: Noción de recurrencia organizada para que la extracción permita poner los caracteres de convexidad capaces de producir los objetivos buscados. El nódulo o bloque puede ser explotado por series sucesivas idénticas.

Tipo E 1: discoide. Es posible producir cuatro tipos de lascas: pseudo puntas Levallois, lascas cuadrangulares, lascas más amplias que largas, y lascas desbordantes (permiten mantenimiento de las convexidades laterales). Los cuatro tipos deben estar necesariamente presentes para permitir la continuidad del débitage. A su vez, las extracciones son predeterminadas y predeterminantes. Puede existir una rotación en el uso de las plataformas de percusión con las superficies de débitage, produciéndose un máximo aprovechamiento de los núcleos, lo cual da como resultado una variabilidad morfológica muy importante entre éstos.

Tipo E 2: piramidal. Pueden producirse lascas alargadas convergentes o no y sobrepasadas para retornar a la estructura del bloque. El mantenimiento de estas categorías permite seguir obteniendo dichos productos. -Es recurrente: cada tipo tiene necesidad de los otros para

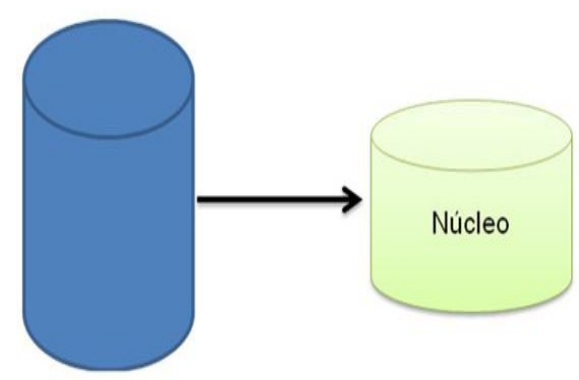

Figura 3. Esquema de la estructura integrada

Figure 3. Integrated structure scheme 
poder producirse.

-Para que produzcan el mismo objetivo, deben cambiar de forma. Se requiere cambiar la estructura para poder mantener los objetivos.

-Son sistemas a la vez cerrados (producciones de algunos tipos obligatorios) y abiertos (cambio de forma). No hay una variabilidad de los métodos sino una variabilidad morfológica debida a la fase de débitage.

Sistema F: Se trata de la preparación de la totalidad del nódulo o bloque para proporcionar una forma con características técnicas particulares, que permite que el tallador conozca con exactitud la morfología y las características de la producción. Se trata del grado mayor de predeterminación.

Tipo F1: Levallois.

Tipo F2: Tecnología de láminas y/o hojas.

En un débitage Levallois: para que la matriz funcione, la forma debe conservar la misma estructura ya que la forma es constitutiva de la estructura (Boëda, 1994; 1997).

Estos sistemas serán tenidos en cuenta en el análisis que se llevara a cabo en este trabajo. Su implementación por los grupos cazadores-recolectores será discutida en base a los distintos lugares donde se encuentra la producción de módulos laminares.

\section{Análisis del modo de producción de módulos laminares a lo largo del Holoceno en el área Interserrana bonaerense.}

La producción de lascas con distintos tamaños y módulos obtenidas a partir de la utilización de la percusión directa/ indirecta y bipolar se ha registrado, en el área Interserrana, desde el Pleistoceno final hasta el Holoceno tardío. Si bien estos tipos de producción están ligados con los objetivos de talla y también con las características de las materias primas, su aprovisionamiento y las actividades realizadas en los distintos sitios arqueológicos, se observa que la producción hojas y de lascas con módulos laminares se encuentra representada en menores proporciones en la mayoría de los sitios investigados.

\section{Ocupaciones del Holoceno temprano y medio: sitio Paso Otero 4.}

Paso Otero 4 forma parte de la localidad arqueológica homónima, ubicada en la cuenca media del río Quequén Grande (partido de Necochea). Es un sitio a cielo abierto donde se recuperó una gran cantidad de materiales óseos y líticos en una superficie excavada de $14 \mathrm{~m}^{2}$ (Gutiérrez et al. 2010, 2011). Las ocupaciones humanas están datadas entre ca. 8900-4600 años AP. Los materiales arqueológicos se distribuyen de manera ininterrumpida a través de la secuencia, aunque se estableció una separación entre niveles inferiores (NI, ca. 8900 a 7700 años AP) y niveles superiores (NS, ca. 7700 a 4600 años $A P)$, en función del registro de fauna extinguida en los primeros (Álvarez et al. 2013).

La materia prima más explotada en el NI es la ortocuarcita GSB con un $n=173$, seguida por ftanita, basalto, y metacuarcita con un ejemplar cada una; por último hay ocho artefactos de materia prima indeterminada. El conjunto artefactual del $\mathrm{NI}$ está compuesto por 27 instrumentos confeccionados sobre ortocuarcita GSB (16 artefactos de formatización sumaria, cinco muescas, dos filos naturales con rastros complementarios, una punta destacada, un raspador, un fragmento de filo e instrumento indeterminado), un núcleo, 132 lascas, 19 productos indiferenciados y cinco productos indiferenciados rodados.

En el NS se continúa con la misma tendencia, donde la ortocuarcita GSB está representada con $n=296$, seguida por ftanita, xilópalo, basalto, y granito con un ejemplar cada una. En este caso fueron 10 los artefactos en los que no se pudo identificar las rocas en las que fueron generados. Se identificaron 43 instrumentos confeccionados sobre ortocuarcita GSB (14 filos naturales con rastros complementarios y 14 artefactos de formatización sumaria, cuatro raederas y fragmentos de filo, dos puntas destacadas y dos muescas, seguido por una punta de proyectil, un instrumento unifacial y un instrumento indeterminado), a los que se suman, un núcleo, un percutor, 214 lascas, 43 productos indiferenciados y ocho productos indiferenciados rodados.

En ambos niveles la roca más explotada fue la ortocuarcita GSB, con un $93,6 \%$ en el $\mathrm{NI}$ y con un $95,7 \%$ para el NS. Además, se utilizó la técnica de percusión directa y bipolar para la obtención de soportes. Se observó un uso similar de módulos laminares en ambos niveles, por un lado, en el $\mathrm{NI}$, de los 30 instrumentos presentes, cuatro presentan este módulo, mientras que de las 76 lascas enteras registradas, sólo seis tienen módulo laminar. Por otro lado, en el NS, se registró un total de 43 instrumentos, donde cuatro presentan este módulo y de las 86 lascas enteras, 13 cuentan con módulo laminar. Si bien es bajo el porcentaje de los productos con módulo laminar, se observa que estuvo presente para la obtención de soportes para la confección de ciertos instrumentos, tanto unifaciales (Figura IV-B) como raederas.

Asimismo, se cuenta al menos con una evidencia de que algunos núcleos (no presentes en el sitio) fueron preparados para poder realizar este tipo de extracciones (Figura IV-A). El análisis de las cadenas operativas permitió inferir la manufactura de instrumentos tanto a partir de artefactos que entraron en avanzado estado de formatización (asignable a las raederas y una punta de proyectil) como a partir de lascas obtenidas de la reducción de núcleos in situ. Los productos residuales fueron utilizados de dos maneras diferentes: por un 
lado, se aprovecharon sus filos naturales y, por otro, se confeccionaron filos por medio de microrretoque y retoque unifacial (Barros et al. 2014). A partir de los desechos de talla se registró que en el sitio se llevaron a cabo actividades de reducción de volumen de los núcleos, producción de potenciales formas base, así como también de manufactura y mantenimiento de instrumentos; estas últimas están más representadas (Barros et al. 2014).

\section{Ocupaciones del Holoceno medio y tardío: Laguna Muscar 2.}

El sitio Laguna Muscar 2 se encuentra próximo a Laguna Muscar 1, en la ciudad de General Lamadrid, provincia de Buenos Aires. Hasta el momento se excavó un área total de $4,75 \mathrm{~m}^{2}$, la cual fue dividida en los sectores $A$ y $B, y$ la cronología se encuentra establecida en 4900 años AP. y 2500 años AP. (Holoceno medio-tardío) (Barros et al. 2018). Se analizó un conjunto artefactual lítico compuesto por 134 elementos (obtenidos en el sector A de la excavación), donde la materia prima más representada es la ortocuarcita GSB con 85 elementos, seguida por la ftanita $(n=38)$, luego la metacuarcita $(n=6)$, la riolita $(n=3)$, la dolomía silicificada y la arenisca ( $n=1$, cada una). Las técnicas utilizadas para la obtención de soportes fueron la percusión directa y la bipolar. Los instrumentos recuperados fueron confeccionados en ortocuarcita GSB $(n=25)$ y ftanita $(n=23)$. Los grupos tipológicos representados sobre los primeros son: siete instrumentos compuestos, siete artefactos de formatización sumaria, cuatro raederas, dos raspadores, cuatro fragmentos de filo y un filo natural con rastros complementarios. Los grupos tipológicos sobre ftanita que han sido recuperados son 12 raspadores, seis instrumentos compuestos, dos instrumentos de formatización sumaria, dos filos naturales con rastros complementarios y un fragmento de filo. En relación al módulo de los instrumentos, el corto muy ancho y el mediano normal son los más representados en ambas materias primas. Se destaca la presencia del modulo laminar en tres casos sobre ortocuarcita GSB y dos en ftanita (Barros et al. 2018).

En relación con las etapas de la cadena operativa, están representadas aquellas relacionadas a la explotación de los núcleos, manufactura de instrumentos y mantenimiento de los mismos. Aunque en el registro sólo se hallaron dos núcleos agotados de ftanita y dolomía silicificada, no se descarta que hayan ingresado núcleos de ortocuarcita GSB. En este sentido, se recuperó una lasca de reactivación de núcleo sobre esta roca.

En base al bajo porcentaje de corteza en la totalidad de la muestra, se estima que tanto los núcleos como otros artefactos entraron al sitio ya descortezados y preparados. En relación con la presencia de módulos laminares, se considera que tanto en ortocuarcita GSB como ftanita, fueron producidos para la realización de instrumentos (Barros et al. 2018).

\section{Ocupaciones del Holoceno tardío: Laguna Seca 2 y Alero La Virgen.}

\section{Laguna Seca 2}

Este sitio se encuentra ubicado a 15 kilómetros al norte de la ciudad de General Lamadrid, provincia de Buenos Aires. Los materiales líticos hallados en superficie comprenden 221 artefactos líticos. Las materias primas representadas en el sitio son ortocuarcita del GSB $(n=124)$, ftanita $(n=54)$, cuarcita $(n=21)$, indeterminadas $(n=7)$, chert $(\mathrm{n}=4)$, dolomía silicificada, riolita, granito y arenisca con dos ítems cada una y por último, solo representada con un artefacto, cuarzo, esquisto y sedimentaria. El conjunto lítico analizado está compuesto por cinco núcleos (3 de ortocuarcita GSB, 1 de ftanita y 1 de cuarcita de Ventania), 45 instrumentos de ortocuarcita GSB (19 raederas, 10 raspadores, cuatro fragmentos de filo, cuatro artefactos de formatización sumaria, cuatro muescas, dos puntas destacadas y dos cuchillos) y 24 de ftanita (14 raspadores, cinco raederas, tres fragmentos de filo, una muesca y una punta destacada) (Barros 2013). En cuanto a los desechos de talla analizados 60 son de ortocuarcita GSB, 29 de ftanita y 12 de cuarcita. Predominan las lascas internas, siendo los talones lisos y lineales los más representados. Se observó que la reducción de la ortocuarcita GSB y de la ftanita se asocia con el objetivo de producción de lascas con módulos medianos, cortos y, en menor medida, laminares. En este sentido, dentro de los instrumentos confeccionados sobre ortocuarcita GSB, solo tres presentan módulo laminar. Mientras que dentro de las lascas enteras también hay tres casos con este módulo. En relación con la ftanita de los 24 instrumentos registrados, tres presentan módulo laminar y dentro de las lascas, tres presentan este módulo. Se observa que estos módulos, fueron seleccionados para la realización de ciertos instrumentos, principalmente raederas (Barros 2013).

Aunque las cadenas operativas de ortocuarcita GSB y ftanita están desarrolladas casi en su totalidad en el sitio, se plantea que el descortezamiento de los núcleos no se efectuó en esta laguna ya que el porcentaje de corteza, tanto en los núcleos como en los desechos de talla, es escaso. En cuanto al tratamiento de los núcleos, se registró un máximo aprovechamiento de la materia prima, lo cual se evidencia en un estado avanzado de reducción, en los tamaños pequeños y en la utilización de técnicas de reducción bipolar.

\section{Alero La Virgen}

El sitio se ubica en el Cerro Largo en las Sierras Bayas, Olavarría. El material lítico se compone de 167 artefactos, pero a diferencia de los otros sitios, en este caso no hay instrumentos. Esta ausencia pudo deberse a distintos factores, entre ellos, la posibilidad de que no fueran abandonados en el sitio, como también que hubiera 
un sesgo debido a la elevada presencia de personas que visitan continuamente el lugar. Las materias primas representadas son ftanita $(n=143)$, cuarcita $(n=23)$ y una roca indeterminada. El conjunto analizado se compone de cuatro núcleos y 163 lascas, de las cuales 68 se presentan enteras. Se constataron dos tipos de producción sobre ftanita, por un lado, lascas con módulo corto y ancho, y por otro lado, lascas de módulo laminar. El primer tipo esta evidenciado en los negativos de los cuatro núcleos recuperados y en parte de la producción, mientras que el de módulo laminar sólo se presenta en los productos de talla $(n=18)$. En este sentido, se puede plantear que existieron núcleos preparados para realizar este tipo de extracción, los cuales pudieron ser descartados en otros lugares, o bien continuar siendo explotados para la obtención de lascas con el otro tipo de módulo (Figura IV C). En el conjunto recuperado también se observa la presencia de lascas de reducción unifacial, bifacial y bipolar. En síntesis, si bien la muestra analizada no es numerosa, se puede concluir que los talladores conocían muy bien cómo adecuar las distintas técnicas a sus objetivos de producción y a la forma como se presentaba la ftanita para poder obtener módulos laminares.

\section{Núcleos del Área del rio Quequén Grande}

Debido tanto al estado de los núcleos, como a su ausencia en los sitios arqueológicos analizados, se consideró necesario contar con una muestra donde se pudieran estudiar núcleos con extracciones laminares para poder entender el desarrollo de su explotación. En este sentido, se analizaron 17 núcleos recolectados en el área arqueológica Zanjón Seco, los cuales fueron estudiados previamente por Martínez (1999).

La totalidad de los núcleos fueron preparados para la extracción de lascas con módulo laminar. En algunos de ellos se presentan escasos negativos, mientras que en otros hay una recurrencia en las extracciones. En relación con la direcciones de los lascados, se observa que 13 de ellos presentan extracciones unidireccionales, seguidos por bidireccionales y multidireccionales con dos casos respectivamente. El mayor porcentaje $(n=14 ; 82,3 \%)$ de los núcleos fueron confeccionados sobre lascas, siendo la superficie ventral de las mismas utilizada como plataforma de percusión (Figura IV D). En los otros 3 casos, el soporte no pudo ser definido debido a su grado de explotación, presentando lascas de preparación y negativos de extracciones que cubren todas las superficies (Figura IV E).

Dentro de los núcleos predominan los de tamaño pequeño, pero también se presentan medianos y grandes. En ciertos núcleos se observa que se ha continuado la explotación para la obtención de lascas con módulos más cortos y anchos. La presencia en un mismo sitio de lascas y productos laminares pueden indicar un proceso operativo idéntico y estar ligado a la noción de recurrencia (Boëda 1997).

\section{Discusión y Conclusión}

El área Interserrana, donde se ubican los sitios Paso Otero 4, Laguna Muscar 2, Laguna Seca 2 y la Localidad Arqueológica Zanjón Seco, cuenta con una escasa presencia de rocas, representados por ciertos afloramientos de toba silicificada, areniscas cuarcíticas y cuarcitas de grano grueso (Politis 1984, entre otros). Las rocas más utilizadas se encuentran distribuidas en los cordones serranos de Tandilia (ortocuarcita GSB, cuarcitas de la Formación Balcarce, ftanita, dolomía silicificada, entre otras) (Barros y Messineo 2004; Bayón et al. 1999; Mazzanti 1997; Messineo et al. 2004; Flegenheimer et al. 1996; Flegenheimer y Bayón 2002; Colombo 2013, entre otros), Ventania (metacuarcita, riolita, ortocuarcita, entre otras) (Oliva et al. 2006; Catella et al. 2010, entre otros) y la costa Atlántica (rocas silíceas, basalto, cuarcita, entre otras) (Bonomo 2005, Bayón y Zavala 1997, entre otros). En cambio, el sitio Alero La Virgen se encuentra ubicado en las Sierras Bayas próximo a las canteras de ftanita que se ubican en el Cerro Largo, en la parte septentrional del cordón serrano de Tandilia.

En relación con los modos de aprovisionamiento de estas distintas rocas a lo largo del Holoceno, se han propuesto modelos que ayudaron a entender cómo fue realizado el mismo (Bayón y Flegenheimer 2004, Bayón et al. 2006, entre otros). En líneas generales, para los momentos tempranos se plantea que los grupos cazadoresrecolectores eran altamente móviles, el aprovisionamiento de rocas era directo y llevaban consigo los artefactos líticos. En cambio, para el Holoceno tardío, varios autores han planteado cambios en la organización de estos

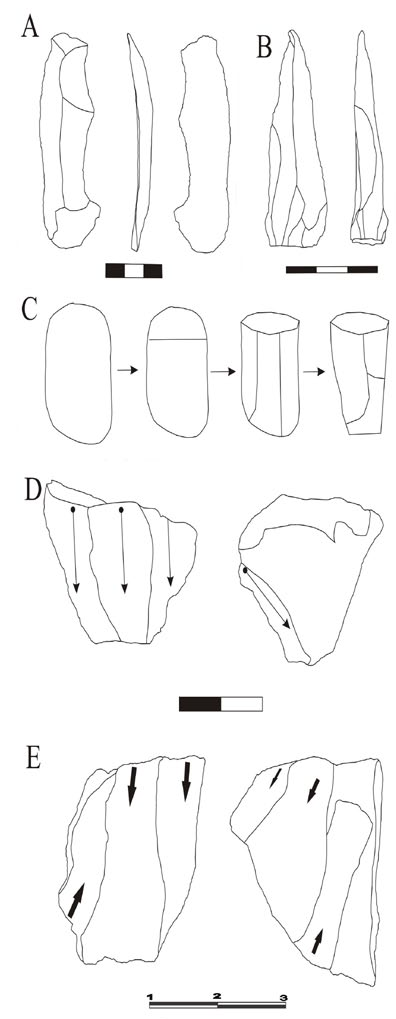

Figura 4. Referencias: IV A. Sitio Paso Otero 4-1246, Nivel: 3,75-3,80. IV B. Sitio Paso Otero 4-904. Nivel: 3,60-3,65. IV C. Esquema de preparación del núcleo de ftanita en el sitio Alero La Virgen. IV D. Núcleo con extracciones laminares cuyo soporte es una lasca. IV E. Núcleo con extracciones laminares donde no se pudo analizar el soporte

Figure 4. References: IV A. Paso Otero 4 site-1246, Level: 3,75-3,80. IV B. Paso Otero 4s site-904. Levell: 3,60-3,65. IV C. Ftanite core from Alero La Virgen prepation shceme. IV D. Flake core with laminar extractions. IV E. Core with laminar extractions (no blank identified) 
grupos, debido principalmente al aumento de la densidad poblacional, lo cual fue acompañado por una reducción de la movilidad (Borrero y Franco 1997, Politis y Madrid 2001, Bayón y Flegenheimer 2004, entre otros). Respecto de la obtención de rocas durante este lapso, se planteó una estrategia de aprovisionamiento de los lugares (sensu Kuhn 1995) la cual generó depósitos de materiales (i e, núcleos, lascas grandes, instrumentos diversos, etc.) que resultaron en una suerte de almacenaje (litificación) de materia prima (Martínez y Mackie 2003-04). Además durante este periodo, se registra un mayor énfasis en el aprovisionamiento indirecto (con respecto a los períodos anteriores) debido al control social de las canteras por grupos determinados (Bayón y Flegenheimer 2004, Colombo y Flegenheimer 2013, Messineo y Barros 2015).

A partir del análisis de las cadenas operativas, realizado en ortocuarcita GSB, se puede observar que para los momentos tempranos, en el sitio Paso Otero 4, esta roca se utilizó entre otras tareas para la obtención de módulos laminares. Para ello, los núcleos fueron preparados con caracteres técnicos específicos. En este sentido, se observa mayor inversión técnica y normalización en este tipo de producción, lo cual está evidenciado a partir de las lascas de preparación para la obtención de estos módulos. A manera de hipótesis se puede proponer que este tipo de producción podría corresponderse con la estructura integrada.

Para el Holoceno medio y tardío, en el sitio Laguna Muscar 2, se observa la presencia de módulos laminares, los cuales han sido seleccionados como soporte de instrumentos sobre ortocuarcita GSB y sobre ftanita. Si bien el porcentaje es bajo, estos soportes fueron utilizados para la confección de instrumentos específicos. En este caso, también se podría estar frente a una estructura integrada. Sin embargo, esto no se puede afirmar taxativamente ya que los mismos solo se encuentran representados bajo la forma de instrumentos.

Para el Holoceno tardío, considerando a los sitios Laguna Seca 2 y Alero La Virgen, se presentan situaciones distintas a las de los otros períodos, ya que sus características están relacionadas y se asemejan a las presentadas en la estructura adicional, del tipo C. Es decir, se seleccionan artefactos por su morfología para ser transformados en núcleos. De todas maneras, siempre se debe tener en cuenta el modo en que se presentan las materias primas, como es el caso de la ftanita, que lo hace bajo la forma de nódulos y bloques de tamaños y formas variables. Algunos de ellos poseen las características técnicas necesarias, y solo se precisa la limpieza de un sector, antes de comenzar la explotación. En este último caso estaríamos en presencia de una estructura adicional tipo D.

El sitio Laguna Seca 2 también presenta módulos laminares de ortocuarcita GSB en bajas proporciones. Debido a lo exiguo del conjunto lítico no es posible determinar si los módulos fueron obtenidos dentro o fuera del sitio. Como en los demás casos, estos soportes fueron seleccionados para la confección de instrumentos.

En el Sitio Alero La Virgen se observa un 10\% de módulos laminares sobre ftanita, lo cual está evidenciado sólo en lascas, porque no se han hallado instrumentos. Se cuenta también con información proveniente de otros sitios, como Calera, Boca de La Sierra, ubicados en el área de Sierras Bayas, donde también existió la explotación de los núcleos para la obtención de este tipo de productos como soporte para la realización de instrumentos (Figura IV C) (Barros et al. 2015, Messineo y Barros 2015).

La presencia de rocas de buena calidad en la mayoría de los sitios analizados indica una importante selección en las canteras. El escaso número de núcleos registrados no permite conocer si los mismos fueron preparados solo para la extracción de lascas con módulo laminar o si luego de algunas extracciones, continuaron siendo explotados para la obtención de otros tipos de módulos. Los núcleos provenientes de sitios superficiales del área del río Quequén Grande evidencian producción de soportes laminares. En las extracciones, ambos ligados con una estructura adicional del tipo C y D.

En síntesis, para los momentos tempranos, a partir de la información de las actividades llevadas a cabo en las canteras del arroyo Diamante (Flegenheimer et al. 1996), conocemos que predominan las lascas nodulares, los núcleos amorfos y los núcleos poco formatizados. La preparación de núcleos de hojas y para la extracción de módulos laminares están representados en baja proporción (Crivelli Montero 1999). Parte de este comportamiento tecnológico, puede asociarse a las técnicas propuestas en una estructura integrada. Esto se basa en que se pudieron seleccionar nódulos o bloques de distintos tamaños, privilegiando los grandes para poder crear las condiciones adecuadas para la explotación laminar (Paulides 2005; Colombo 2013). Luego, parte de los núcleos preparados pudieron ser reducidos en las canteras, logrando la obtención de soportes que, en algunos casos pudieron ser transformados en instrumentos, y haber salido de las canteras bajo estas formas (soportes y/o instrumentos). En otros casos, los talladores llevaron los núcleos preparados fuera de las canteras. La falta de estos núcleos en los sitios puede deberse a que pudieron seguir siendo explotados para la obtención de otros tipos de módulos. Esto puede estar avalado por la presencia de instrumentos con módulo laminar y en la ausencia de núcleos con este tipo de negativos. Es importante mencionar que estos tipos de soportes fueron seleccionados para la confección de diferentes instrumentos, siendo las raederas, los más representados en los conjuntos pampeanos. Para los momentos del Holoceno tardío, se presentan cambios en relación con el abastecimiento, los cuales pueden relacionarse con ciertos factores como el aumento demográfico, los grupos moviéndose en sectores más 
acotados y el acceso restringido a las canteras (Bayón y Flegenheimer 2004, Colombo y Flegenheimer 2003, Messineo y Barros 2015, entre otros). Estos cambios, también se dieron en el modo de producción, pasando desde una estructura integrada a una adicional. De esta manera, la producción de módulos laminares estuvo ligada a la obtención de soportes para la fabricación de ciertos tipo de instrumento. La baja representación en distintos sitios de la región a lo largo del Holoceno, podría estar enmascarada por las modificaciones que sufre el material desde que es utilizado para la fabricación de soportes laminares, hasta que es descartado. En este sentido podría darse que elementos que estuvieran asociados a la producción laminar luego de sucesivas transformaciones sean finalmente abandonados con morfologías que no permitan inferir su origen laminar. Es necesario conocer su representatividad en todas las áreas de la región pampeana, para lograr un mayor entendimiento de su producción y elección. Es un modo de producción que describe el conocimiento técnico de los grupos que habitaban el área Interserrana de la región pampeana, que aunque se encuentra poco representado fue una opción utilizada para la fabricación de soportes.

Olavarría, 7 de julio de 2017

\section{Agradecimientos}

A Manuel Carrera Aizpitarte y Florencia Santos Valero por las sugerencias vertidas al trabajo. A Gustavo Martínez, María Gutiérrez, Pablo Messineo, Cristian Kaufmann, y Jonhatan Bellinzoni por permitirme usar los datos generados en trabajos en conjunto. A Dan Rafuse por la realización del mapa. Al Grupo Amigos del Museo de Ciencias Naturales "Gesué P. Noseda" del Club de Pesca de Lobería, principalmente a Beatriz Baglivo, por su buena predisposición. A los evaluadores del trabajo por sus exhaustivos comentarios que mejoraron sustancialmente el trabajo. Las investigaciones fueron financiadas por la ANPCYT (PICT 2010-1415 y PICT 2014-2070) y CONICET (PIP N 0414); Mission Archéologique Française en Argentine, dirigido por Rodolphe Hoguin (año 2014). Este artículo es una producción de la Unidad Ejecutora INCUAPA-CONICET (Investigaciones Arqueológicas y Paleontológicas del Cuaternario Pampeano) dirigido por el Dr. Gustavo G. Politis. Las opiniones vertidas en el presente trabajo son de mi absoluta responsabilidad.

\section{Bibliografía}

Aschero, C. 1975. Ensayo para una clasificación morfológica de artefactos líticos aplicada a estudios tipológicos comparativos. Informe al CONICET. Ms.

1983. Ensayo para una clasificación morfológica de artefactos líticos. Apéndice A y B. Cátedra de Ergología y Tecnología. Facultad de Filosofía y Letras. Universidad de Buenos Aires. Ms.
Álvarez, M. C., A. P. Alcaráz, M. A. Gutiérrez y G. Martínez 2013. Análisis zooarqueológico del sitio Paso Otero 4 (partido de Necochea). Aportes a la discusión de modelos de subsistencia de la región pampeana. Intersecciones en Antropología 14: 383-398.

Barros, M. P. 2013. El estudio del aprovisionamiento y la circulación de materias primas en el sitio Laguna Seca 2 (Partido de General Lamadrid, provincia de Buenos Aires). Volumen especial de Arqueología de la Región Pampeana. Revista del Museo de La Plata. Vol.: 13 pp. 185-197 . ISSN 0376-2149

Barros, P. y P. G. Messineo. 2004. Identificación y aprovisionamiento de chert o ftanita en la cuenca superior del Arroyo Tapalqué. Estudios Atacameños 28: 87-103.

Barros, M. P.; Martínez, G. y M. A. Gutiérrez. 2014. Análisis de los materiales líticos del sitio Paso Otero 4 (Partido de Necochea, provincia de Buenos Aires). Avances en el conocimiento de las estrategias tecnológicas en el curso medio del río Quequén Grande para el Pleistoceno tardío-Holoceno. Relaciones de la Sociedad Argentina de Antropología XXXIX (1):119-144.

Barros, M. P., P. G. Messineo y M. J. Colantonio. 2015. Exploitation of lithic raw material in the Northwestern sector of Tandilia and the stone circulation in the center of the Humid Pampas subregion. Quaternary International 375: 99-112.

Barros, M. P., Bellinzoni, J. y C. Kaufmann. 2018. Análisis de los instrumentos líticos de los sitios Laguna La Redonda y Laguna Muscar 2 (Partido de Gral. Lamadrid, provincia de Buenos Aires). Intersecciones en Antropología 19(2). En prensa.

Bayón, C. y C. Zavala. 1997. Coastal Sites in Southern Buenos Aires: A Review of "Piedras Quebradas". Quaternary of South America Antartic Peninsula 10: 229-253.

Bayón, C. y N. Flegenheirmer. 2004. Cambio de planes a través del tiempo para el traslado de roca en la pampa bonaerense. Estudios Atacameños 28: 59-70.

Bayón, C., N. Flegenheirmer, M. Valente y A. Pupio. 1999. Dime cómo eres y te diré de dónde vienes: la procedencia de rocas cuarcíticas en la Región Pampeana. Relaciones de la Sociedad Argentina de Antropología XXIV: 187-235.

Bayón, C., Flegenheimer, N. y A. Pupio. 2006. Planes sociales en el abastecimiento y traslado de roca en la Pampa Bonaerense en el Holoceno Temprano y Tardío. Relaciones de la Sociedad Argentina de Antropología XXXI: 19-45. 
Bellelli, C., A. Guraieb y J. García 1985-87. Propuesta para el análisis y procesamiento por computadora de desechos de talla lítica (DELCO -Desechos Líticos Computarizados). Arqueología 2(1): 36-53.

Boëda, E. 1994. Le concept Levallois: variabilité des méthodes. París, C.N.R.S. Editions (Monographie du C.R.A., 9), París.

1997. Technogenèse de systèmes de production lithique au Paléolithique inférieur et moyen en Europe occidentale et au Proche-Orient. Thèse d'Habilitation à diriger des recherches, Université de Paris X-Nanterre.

2005. Paléo-technologie ou anthropologie des Techniques?. Arobase, volumen 1, pp : 46-64.

Boëda, E., J.-M. Geneste y L. Meigen 1990. Identification des chaînes opératoires lithiques au Paléolithique inférieur et moyen. Paléo 2: 43-80.

Bonomo, M. 2005. Costeando las llanuras. Arqueología del litoral marítimo pampeano. Buenos Aires, Colección de Tesis Doctorales, Sociedad Argentina de Antropología.

Borrero L. y N. Franco. 1997. Early Patagonian huntergatherers: Subsistence and technology. Journal of Anthropological Research 53: 219-239.

Catella, L., Moirano, J. y F. Oliva. 2010. Disponibilidad de materias primas líticas y su importancia para el análisis del uso del espacio y la organización de la tecnología en sociedades cazadoras recolectoras. En M. Berón, L. Luna, M. Bonomo, C. Montalvo, C. Aranda y M. Carrera Aizpitarte (eds.), Mamul Mapü: pasado y presente. Perspectivas de la arqueología pampeana a comienzos del tercer milenio, Tomo II: 239-254. Ayacucho, Editoriales del Espinillo.

Colombo, M. 2013. Los cazadores y recolectores y sus rocas. La obtención de materias primas líticas vista desde las canteras arqueológicas del centro de Tandilia. Tesis doctoral inédita, Facultad de Ciencias Naturales y Museo, Universidad de La Plata, La Plata.

Colombo, M. y N. Flegenheimer. 2013. La elección de rocas de colores por los pobladores tempranos de la región pampeana (Buenos Aires, Argentina): Nuevas consideraciones desde las canteras. Boletín del Museo Chileno de Arte Precolombino, 18 (1), 125-137.

Crivelli Montero, E. 1999. Comentario al artículo "Dime cómo eres y te diré de dónde vienes: procedencia de rocas cuarcíticas en la Region Pampeana" de C. Bayón, A. Pupio, M. Valente y N. Flegenheimer. Relaciones de la Sociedad Argentina de Antropología; tomo 24: 231-232

Flegenheimer, N. y C. Bayón. 2002. Cómo, Cuándo y
Dónde? Estrategias de abastecimiento lítico en la Pampa Bonaerense. En D. Mazzanti, M. Berón y F. Oliva (eds.), Del Mar a los Salitrales, Diez Mil Años de Historia Pampeana en el Umbral del Tercer Milenio: 231-241. Mar del Plata, Facultad de Humanidades. Universidad Nacional de Mar del Plata.

Flegenheimer, N., S. Kain, M. Zárate y A. Barna. 1996. Aprovisionamiento de cuarcitas en Tandilia, las canteras del Arroyo Diamante. Arqueología 6: 117-141. Buenos Aires. Revista de la Sección Prehistoria, ICA-UBA.

Fogaça, E. y E. Boëda 2006. A Antropología das técnicas e o povoamento da América do Sul Pré-histórica. Habitus 4(2): 673-684.

Geneste, J.M. 1991. L'approvisionnement en matières premières dans les systèmes de production lithique: la dimension spatiale de la technologie. In: Tecnología y cadenas operativas líticas. Reunión internacional, 15-18 enero de 1991: 1- 36.

Gutiérrez, M. A., G. Martínez, H. Luchsinger, M. C. Álvarez y M. P. Barros. 2010. Investigaciones arqueológicas y geoarqueológicas preliminares en el sitio Paso Otero 4 (Partido de Necochea). En M. Berón, L. Luna, M. Bonomo, C. Montalvo, C. Aranda y M. Carrera Aizpitarte (eds.), Mamul Mapü: pasado y presente. Perspectivas de la arqueología pampeana a comienzos del tercer milenio, Tomo II: 69-84. Ayacucho, Editorial Libros del Espinillo.

Gutiérrez, M. A., G. Martínez, H. Luchsinger, S. Grill, A. Zucol, G. Hassan, M. P. Barros, C. Kaufmann y M. C. Álvarez. 2011. Paleoenvironments in the Paso Otero locality during late Pleistocene-Holocene (pampean region, Argentina): an interdisciplinary approach. Quaternary International 245: 37-47.

Inizan, M.-L., M. Reduron, H. Roche y J. Tixier 1995. Technologie de la pierre taillée. Meudon, CREP.

Kuhn, S., 1995. Mousterian lithic technology. An ecological perspective. Princeton University Press, Princeton

Madrazo, G. 1972. Arqueología de Lobería y Salliqueló (Pcia. de Buenos Aires). Etnia: 15: 1-18. Olavarría.

Madrazo, G. 1973. Síntesis de la Arqueología Pampeana. Etnía 17: 13-25. Olavarría.

Madrazo, G. 1979. Los cazadores de larga distancia de la Región Pampeana. Prehistoria Bonaerense: 13-67. Olavarría.

Martínez, G. A. 1999. Tecnología, subsistencia y asentamiento en el curso medio del Río Quequén Grande: un enfoque arqueológico. Tesis Doctoral Inédita, Facultad 
de Ciencias Naturales y Museo, Universidad Nacional de La Plata, La Plata.

Martínez, G. y Q. Mackie. 2003-04. Late Holocene human occupation of the QuequénGrandeRiver valley bottom: settlement systems and an example of a built enviroment in the Argentine Pampas. En L. Barham (ed.), Before farming: the archaeology and anthropology of huntergatherers: 178-202. Bristol. Inglaterra.

Mazzanti, D.1997. Excavaciones arqueológicas en el sitio Cueva Tixi, Buenos Aires, Argentina. Latin American Antiquity 8: 55-62.

Messineo, P. G., M. P. Barros, D. Poiré y L. Gómez Peral. 2004. Características litológicas de los niveles de cherto ftanita en la Sierras Bayas (Partido de Olavarría, Provincia de Buenos Aires). En G. Martínez, M. Gutiérrez, R. Curtoni, M. Berón y P. Madrid (eds.), Aproximaciones Contemporáneas a la Arqueología Pampeana. Perspectivas Teóricas, Metodológicas, Analíticas y Casos de Estudio: 307- 319. UNICEN-FACSO, Olavarría.

Messineo, P. G. y M. P. Barros. 2015. Lithic raw materials and modes of exploitation in quarries and workshops from the center of the Pampean grassland of Argentina. Lithic Technology 40 (1): 3-20.

Nami, H. 1999. Variaciones sobre rocas cuarcíticas (pp: 223-229), comentario al artículo: Dime cómo eres y te diré de dónde vienes: Procedencia de rocas cuarcíticas en la región pampeana. Relaciones de la Sociedad Argentina de Antropología XXIV, pp. 187-222.

Oliva, F., Ávila, J.D., Ayuso, M. P., Catella, L. y M. Gallego. 2006. Investigaciones arqueológicas en la cuenca del arroyo Chasicó y llanura adyacente, sudoeste de la provincia de Buenos Aires. Comenchingonia. Revista de Arqueología 9: 123-139.

Paulides, L. 2005. Formando rocas: la organización tecnológica del complejo de canteras de arroyo El Diamante, partido de Benito Juárez (provincia de Buenos Aires). Tesis de Licenciatura inédita. Facultad de Filosofía y Letras, Universidad de Buenos Aires, Buenos Aires

Pelegrin, J. 1995. Technologie lithique - Le Châtelperronien de Roc-de-Combe (Lot) et de La Côte (Dordogne). París, C.N.R.S Éditions, Cahiers du Quaternaire, 20.

Perlès, C. 1991. Économie des matières premières et économie du débitage: deux conceptions opposées? En : 25 ans d'études technologiques en préhistoire: bilan et perspectives. (Actes des Xle rencontres internationales d'Archéologie et d'Histoire d'Antibes): 35-45.

Politis, G. 1984. Arqueología del área Interserrana Bonaerense. Tesis Doctoral no publicada. Facultad de Ciencias Naturales y Museo, Universidad de La Plata. La Plata.

Politis, G. y P. Madrid, 2001. Arqueología pampeana: Estado actual y perspectivas. En Historia argentina prehispánica, E. Berberián y A. Nielsen (Eds.), T. II: 737813. Editorial Brujas, Córdoba. 